Proceedings of the 1998 IEEE

International Conference on Control Applications

Trieste, Italy 1-4 September 1998

\title{
Position-dependent Friction Compensation for Ballscrew Tables
}

\author{
Pai-Yi Huang*, Yung-Yaw Chen* and Min-Shin Chen** \\ "Department of Electric Engineering, National Taiwan University, Taipei, Taiwan, R.O.C \\ **Department of Mechanical Engineering, National Taiwan University, Taipei, Taiwan, R.O.C \\ Email: pyi@ipment.ee.ntu.edu.tw
}

\begin{abstract}
This paper describes a compensation scheme for positional-dependent friction in ballscrew-type tables. Based on the observations of the experimental data, the friction of the ball-screw table exhibits periodical behovior with varying amplitudes upon different velocities. The friction profile function $f_{s}(x)$ can be determined by measuring the static friction in each position. The parameter of amplitude for scaling the friction profile is calculated in real time for friction compensation. This formulation is then applied to the friction observer developed by Hassig and Friedland. The convergence of the modified friction compensator is proved and the simulation results demonstrate the ability of the proposed method.
\end{abstract}

\section{Introduction}

Frictions exist unavoidably in mechanical systems with contact. The nonlinear behaviors induced by friction would limit the performance of the controlled system. With the position resolutions increasing, good compensations for these phenomena are becoming more and more important.

A survey of a number of friction compensation methods has recently appeared [1]. The friction models [2-5] are all focus on velocity-dependent characteristics. The important topics for the friction models are static (stick) friction, Coulomb friction, stick-slip phenomena, viscous friction and friction memory. Most of the friction models are devoted to the above characteristics The position-dependent behaviors are often ignored or conquered by designing with large model adaptation gains.

For position-dependent friction part, an adaptive pulse width controller is proposed in [6]. At final stage of the positioning, the controller would adaptively change the width and magnitude of the output pulses with respect to current measured error. In this way, the problem of position-dependent friction is solved. Tomizuka [7] had proposed another interesting approach based on repetitive control. The scheme is applied for repetitive tasks. With enough computer memory, the position-dependent information are recorded and adjusted repetitively. After a few learning cycles, the controller performs well for repetitive tasks.

The friction compensation method proposed in this paper is based on experimental observation. The friction phenomena existed in ballscrew type $X Y$ table is perceived with some hidden relations. A positiondependent model is then proposed after the observation. Computer simulations show the ability of the proposed scheme.

\section{0-7803-4104-X/98/\$10.00 @1998 IEEE}




\section{Position-dependent Friction Model}

Phillips and Ballou [8] described the detailed friction modeling of the General Electric GP132 industrial robot. They found that due to the positiondependent friction, it is difficult to maintain a small, constant velocity with link 3 of the robot. They also mentioned that the position-dependent friction has three characteristics; amplitude, period and phase. The first two are easy to identify, but the phase of the actual friction was not constant but varied randomly between manipulator movements. Adaptive friction compensation in some form of phase tracker would require that the reference signal be available for many cycles. However, in real situations, this may not be a reasonable assumption.

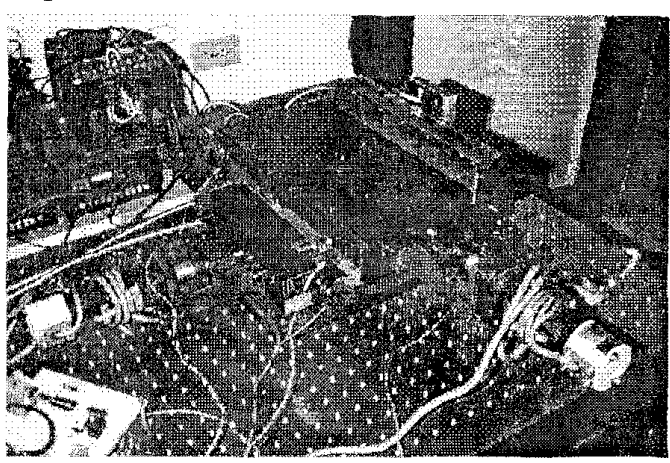

Figure 1. The XY table in experiment

Similar experiments are done on a ballscrew type $\mathrm{XY}$ table. The table driven by DC motor in Figure 1 was made by NSK. Ideally when a constant voltage is applied to the DC motor, the table would move at a constant speed. Due to the inference of the position-dependent friction, the speed would vary with time. Applying different voltages and collecting the measured data draws figure 2 . The horizontal axis is time and the vertical axis is velocity. From this figure, the characteristics of friction in constant voltage are both frequency-vary and time-vary. There are no gravity loads as in [8], thus the data is more simple to analysis.

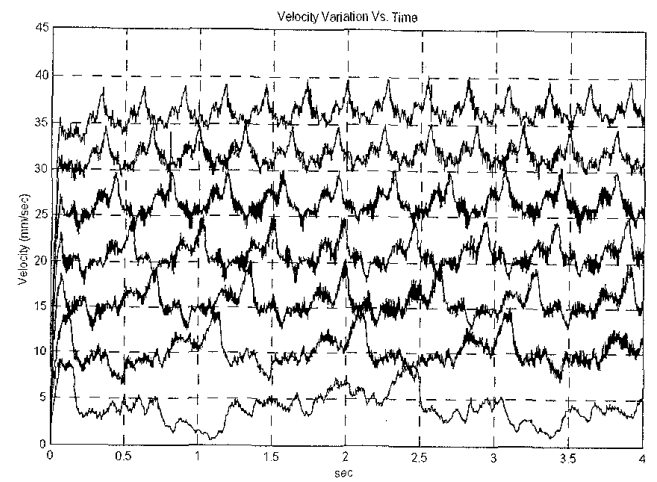

Figure 2. Velocities Vs Time

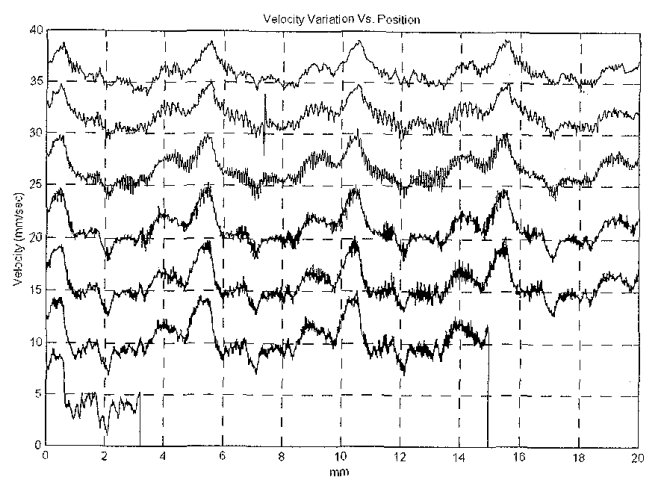

Figure 3. Velocities Vs Position

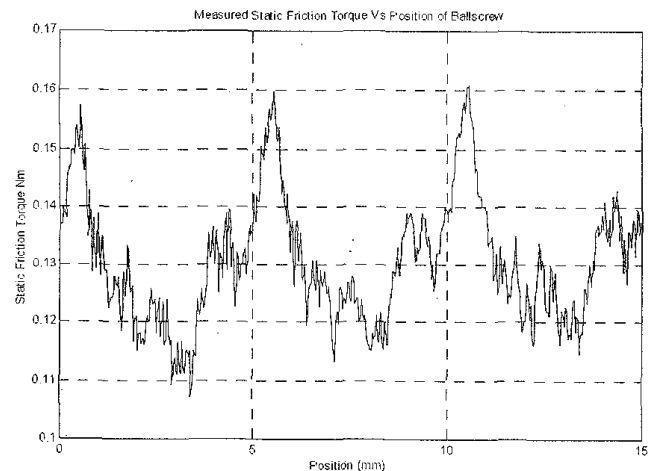

Figure 4. Static Friction Measured Vs Positions

The sample-time data in Figure 2 are interpolated to transform the relation from time to position. The result is shown in Figure 3. The behaviors of the friction can now easily be seen. The pattern of position-dependent friction repeats itself in $5 \mathrm{~mm}$. This distance is equal to the pitch of the ballscrew. The amplitudes are varying 
when different voltages applied, but the shape of the friction remains the same. Figure 4 is the measured static friction force (torque) vs. positions. The friction pattern observed is the same as those in Figure 3.

A friction model for ballscrew table under study is proposed after the observation. By noticing that the shape of the position-dependent friction is not changing when the moving velocities varying, but the amplitude do. The total friction effect is the function of velocity $v$ and position $x$. That is

Friction Force: $F_{r}(x, v)$.

They can be decomposed into two parts, one for velocity-dependent part and one for position-dependent part.

$$
F_{r}(x, v)=F_{r x}(x) \cdot F_{r}(v) .
$$

where $x$ is the position, $v$ is velocity. The positiondependent part can further separate into two part.

$$
F_{r x}(x)=a \cdot f_{s}(x)
$$

Where $f_{s}(x)$ is the shape function of the positiondependent friction and $a$ is a scaling factor. In order to reduce the effect of the phase problem, the shape function can be obtained from the static friction measurement in each position. In [8], a method for obtaining the static (breakaway) friction is described.

We may not know the time delay of the friction effect at each velocity, but from the static friction measurement we may locate the exact position that nonlinearity would occur. The phase problem is due to the difficulties to estimate the phase or delay on line. In our formulation, the static friction measured is surely in phase. Thus, the phase is not a problem.

\section{Analysis}

Hassig and Friedland [9] developed a nonlinear Coulomb friction observer, and a series of papers have been published. In this part of the paper, we would modify the formulation in [9] to cope with the position- dependent friction. The Coulomb friction is in the form

$$
f(v, b)=b \cdot \operatorname{sgn}(v)
$$

where $b$ is also a constant. This term represented the velocity-dependent part $F_{r v}$ in equation (2). After combined with position-dependent term $F_{r x}$, the total friction term becomes

$F_{r}(x, v, c)=f(v, b) \cdot F_{r x}(x)=c \cdot \operatorname{sgn}(v) \cdot f_{s}(x)$,

where $c=a \cdot b$. The purpose of the following derivation is to find a friction parameter observer for estimating parameter $\mathcal{C}$.

The dynamics of the process under study is assumed to be formulated by

$$
\dot{v}=-F_{r}(x, v, c)+w,
$$

where $w$ is the force due to all control forces other than friction. An estimator for undetermined parameter $c$ is the same in [9] with the from

$$
\hat{c}=z-k|v|^{\mu}
$$

where $\hat{c}$ is the estimation of $c$, and $k>0$ and $\mu>0$ are design parameters. The variable $\mathrm{z}$ is given by

$$
\dot{z}=k \mu|v|^{\mu-1}\left[w-F_{r}(x, v, c)\right] \cdot \operatorname{sgn}(v)
$$

The followings are the proof for the convergence of the algorithm. Letting error of the estimator be

$$
e=c-\hat{c} .
$$

Differentiate both part, we have

$$
\begin{aligned}
& \dot{e}=-\dot{\hat{c}} \\
& =-\dot{z}+k \mu|v|^{\mu-1} \dot{v} \cdot \operatorname{sgn}(v) \\
& =k \mu|v|^{\mu-1} \operatorname{sgn}(v)\left[\dot{v}-w+F_{r}(x, v, \hat{c})\right],
\end{aligned}
$$

by using system dynamic in (6) the equation becomes

$$
\begin{aligned}
\dot{e} & =-k \mu|v|^{\mu-1} \operatorname{sgn}(v)\left[F_{r}(x, v, c)-F_{r}(x, v, \hat{c})\right](13) \\
& =-k \mu|v|^{\mu-1} \operatorname{sgn}(v)\left[(c-\hat{c}) \cdot \operatorname{sgn}(v) \cdot f_{s}(x)\right](14)
\end{aligned}
$$




$$
=-k \mu|v|^{\mu-1} f_{S}(x) \cdot e,
$$

because the static friction is positive every where in $x$, the term $k \mu|y|^{\mu-1} \cdot f_{s}(x)$ is positive, too. the error $e$ of the estimator can converge to zero.

In the case of unsymmetry friction in different moving direction, two friction pattern functions can be defined. One for forward table movement and the other for backward. The result of the convergence can still work.

\section{Simulation Study}

The simulation was performed on a second order system [8].

$$
\begin{aligned}
& \dot{x}=v \\
& \dot{v}=-F_{r}(x, v, c)+u
\end{aligned}
$$

where $F_{r}(x, v, c)=c \cdot \operatorname{sgn}(v) \cdot f_{s}(x)$.

In this example the control law is chosen the same as in [9];

$$
u=-200\left(x-x_{d}\right)-20 \cdot v
$$

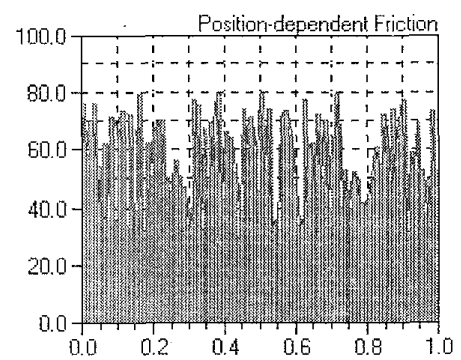

Figure 5. Friction Pattern Generated

The friction pattern in the case of ballscrew table is a periodic one. In computer simulation, $f_{s}(x)$ is randomly generated. Without loss generality, the friction pattern is assumed to happen periodically in $1 \mathrm{~m}$ distance in simulation. The $1 \mathrm{~m}$ distance is then divided into 100 parts and the value of the friction in each part is taken between $30 \sim 80 \mathrm{~N}$. The results of the generated friction pattern is shown in Figure 5
The simulation is taken on Matlab software. Two compensation schemes are studied, one for friction observer [9] and the other for proposed scheme. The design parameter value $\mu$ and $\mathrm{k}$ in equation (7) is defined as follows

$$
\mu=2 \text { and } k=1
$$

A square wave reference signal with $1.1 \mathrm{~m}$ amplitude, slightly greater than pattern period $1 \mathrm{~m}$, is chosen. The Figure 6 shows the simulation while using friction observer, and in Figure 7 shows the results of the proposed method. The dash-dot lines in the upper part of Figure 6 and Figure 7 represent ideal responses and lower part represent the true frictions.

Observing the lower part of Figure 6. The friction observer can not estimate the true position-dependent friction. Only the main value of the frictions is found.
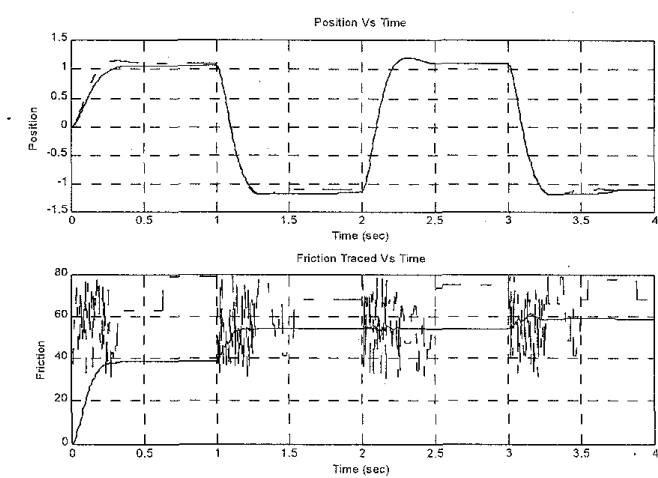

Figure 6. Constant Friction Compensation
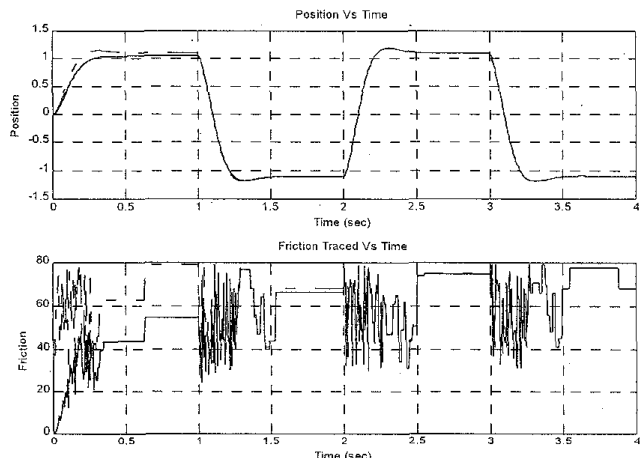

Figure 7. Position-dependent Compensation 
The position response indicated that tracking errors are enlarged at each switching of the reference signal. For the Figure 7 part, the proposed method worked well. The true friction can be traced almost at second cycle. At the start, the position error is large, but soon it is converged to zero.

\section{Conclusion}

A model for position-dependent friction on ballscrew type table is proposed. Then combined with the nonlinear friction observer [9], an extended version for position-dependent friction observer is developed. The convergence of the algorithm is based on the positive property of the friction itself. Computer simulations show the superiority of the proposed method. Further works will be the implementation of the method to real ballscrew systems, and extending this idea to more general friction models.

\section{Reference}

[1] B. Armstrong-Helouvry, P. Dupont, and C. Canudas de Wit, "Survey of Models, Analysis Tools, and Compensation Methods for Control of Machines with Friction," Automatica, vol.30, no.7, pp. 10831138, July 1994.

[2] Dahl, P. R., "A Solid Friction Model," AFO 469567-C-0158, Aerospace Corporation, EI Segundo, CA. 1968.

[3] Karnopp, D., "Computer Simulation of Stick-slip Friction in Mechanical Dynamic Systems," Transactions of the ASME Journal of Dynamic Systems, Measurement and Control, Vol. 107, pp. 100-103, 1985.

[4] Armstrong, B., Control of Machines with Friction, Kluwer Academic Publishers. 1991.

[5] Canudas, C., Olsson, H., Astrom, K. J., and Lischinsky, P. "A New Model for Control of
Systems with Friction," IEEE Transactions on Automatic Control. Vol. 40, No. 3, pp. 419-425. 1995.

[6] Yang, S., and Tomizuka, M., 1988, "Adaptive Pulse Width Control for Precise Positioning under the Inference of Stiction and Coulomb Friction," Transactions of the ASME Journal of Dynamic Systems, Measurement, and Control, Vol. 110, pp. $221-227,1988$

[7] Tomizuka, M., Tsao, T. C., and Chew, K. K., "Analysis and Synthesis of Discrete-time Repetitive Controllers," Transactions of the ASME Journal of Dynamic Systems, Measurement and Control, Vol. 111, pp. 353-358, 1989.

[8] Phillips, S., M., and Ballou, K., R. "Friction Modeling and Compensation for an Industrial Robot," Journal of Robotic System 10(7), pp. 947971, 1993.

[9] Hassig, D. A., and Friedland, B., 1991, "On the Modeling and Simulation of Friction," Transactions of the ASME Journal of Dynamic Systems, Measurement and Control, Vol. 113, pp. 354-362. 